\title{
Book Titled Nature-Inspired Networking: Theory and Applications: An Introduction
}

\author{
Phan Cong Vinh \\ Nguyen Tat Thanh University \\ 300A Nguyen Tat Thanh street, Ward 13, District 4, Ho Chi Minh City, Vietnam
}

\section{Abstract}

Nature-Inspired Networking: Theory and Applications contains nine original, peer-reviewed chapters reporting on new developments of interest to the nature-inspired networking/computing communities. All chapters contain remarkable topics of nature-inspired networking (NiN), from theory to applications for NiN based on rigorous interdisciplinary approaches in which theoretical contributions have been formally stated and justified, and practical applications have been based on their firm, formal basis. The book is a reference for readers who already have a basic understanding of networking and are now ready to know how to use rigorous approaches to develop networking that is inspired by nature. Chapters were carefully selected to, on one hand, cover a broad spectrum of formal and practical aspects and, on the other hand, achieve as much as possible in a self-contained book.

Received on 16 April 2018; accepted on 16 April 2018; published on 18 June 2018

Keywords: NiN, Nature-inspired networking, Nature-inspiration

Copyright (C) 2018 Phan Cong Vinh, licensed to EAI. This is an open access article distributed under the terms of the Creative Commons Attribution license (http: / / creativecommons . org/licenses/by/3.0/), which permits unlimited use, distribution and reproduction in any medium so long as the original work is properly cited.

doi:10.4108/ai.18-6-2018.154821

\section{Introduction}

A new networking paradigm, which is seen as a cuttingedge approach to networks, is currently a priority research area: nature-inspired networking $(\mathrm{NiN})$, which is inspired from nature such as biological, social, and physical phenomena.

The book [1] is a place for highly original ideas about how the nature is going to shape networking systems of the future. Hence, it focuses on theory and applications, which encompass rigorous approaches and cuttingedge solutions that take inspiration from nature for the development of novel problem solving techniques. To this end, we will take advantage of formal engineering methods and establish in this book formal and practical aspects of $\mathrm{NiN}$ to achieve foundations and practice of NiN.

The book (cover of the book at Fig. 1) is a reference for readers who already have a basic understanding of networking and are now ready to know how to use rigorous approaches to develop networking that is inspired by nature. Hence, the book includes both

*Corresponding author. Email: pcvinh@ntt.edu.vn theoretical contributions and reports on applications. To keep a reasonable tradeoff between theoretical and practical issues, chapters were carefully selected to, on one hand, cover a broad spectrum of formal and practical aspects and, on the other hand, achieve as much as possible in a self-contained book.

Formal and practical aspects are presented in a straightforward fashion by discussing in detail the necessary components and briefly touching on the more advanced components. Therefore, theory and applications demonstrating how to use the formal engineering methods for $\mathrm{NiN}$ will be described using sound judgment and reasonable justifications.

This book, with chapters contributed by prominent researchers from academia and industry, will serve as a technical guide and reference material for engineers, scientists, practitioners, and researchers by providing them with state-of-the-art research findings and future opportunities and trends. These contributions include state-of-the-art architectures, protocols, technologies, and applications in NiN. In particular, the book covers existing and emerging research issues in $\mathrm{NiN}$. The book has nine chapters addressing various topics 


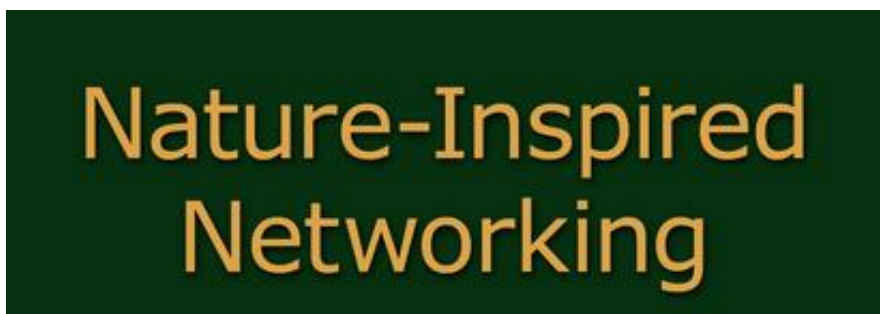

Theory and Applications

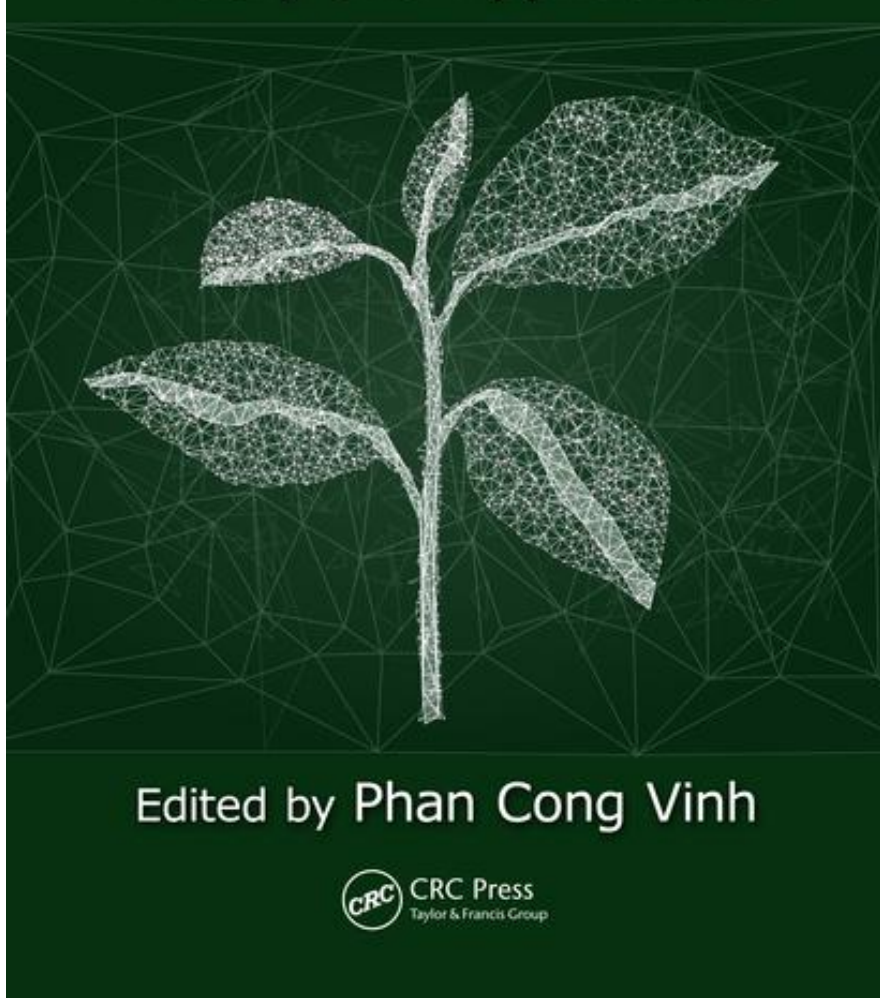

Figure 1. Cover of the book

from theory to applications of $\mathrm{NiN}$ based on rigorous interdisciplinary approaches.

\section{Contents of the Book}

Chapter 1 by P.C. Vinh considers self- ${ }^{*}$ as a foundation for autonomic computing. The notion of autonomic systems (ASs) and self- ${ }^{*}$ serves as a basis on which to build the intuition about algebraic aspects of ASs in general. In this chapter author specifies ASs and self- $^{*}$ and then moves on to consider some universal constructions such as products, coproducts, curried self- $^{*}$ actions, finite limits, colimits of ASs, and monoids of self- ${ }^{*}$ actions. All of this material is taken as an investigation of the algebraic aspects of ASs.

Chapter 2 by F. Chiti, E. Dei, and R. Fantacci reviews the most relevant bio-inspired routing and clustering approaches with a special focus on vehicular scenarios. The underlying concept of mobile group management is adopted to select the more suited schemes, among which are the ant or bee colonies organization and the bacteria life cycle. Finally, the practical issues related to protocol design are investigated by relying on bacterial quorum sensing mechanism, which seems to be a promising approach to design decentralized and flexible joint clustering and routing schemes, while achieving a full context awareness.

Chapter 3 by J. Sánchez-García, J. M. García-Campos, D. G. Reina, S. L. Toral, and F. Barrero indicates that nowadays nature-inspired algorithms can be applied to many fields and provide optimal or pseudo-optimal solutions to many different optimization problems. Disaster scenarios, which present a highly dynamic nature, are one of these areas of application that can benefit from nature-inspired algorithms. Specifically, mobile ad hoc networks which are envisioned to be deployed in disaster scenarios present several aspects that need to be addressed with flexibility, accuracy, and efficiency. Nature-inspired algorithms present the required characteristics for efficiently dealing with several optimization problems related to multi-hop ad hoc networks in disaster scenarios. The authors propose the chapter for gathering and organizing the information about current works in this field.

Chapter 4 by A.M. Alberti, R.d.Rosa Righi, M.A.F. Casaroli, and D. Singh focuses on exploring the synergies, dependencies, and common requirements between the future Internet and distributed systems, discussing the relationships between both research areas. It provides a detailed description of NovaGenesis and newer challenges in distributed systems (evolutionary and revolutionary). Furthermore, it addresses communication model, message passing, naming, identification, addressing, and locating issues, as well as approaches for modeling distributed systems and communication protocols under the concepts of NovaGenesis. With this scope, the chapter will naturally touch on other research areas, including cloud-based storage and networking, network management, virtualization, Internet of things, operating systems, autonomic networking, context-awareness, evolutionary networking, and even hardware solutions. The contributions permeate not only networking protocols and architectures, but also distributed systems and software engineering.

Chapter 5 by Ratneshwer and V. Kushwaha indicates that while collective intelligence is considered as a powerful approach to enhance decision capabilities, very few attempts have been made to utilize its potential in the computer network domain. This chapter is intended to provide the general overview of collective intelligence with a discussion on how it can be effectively applied in different areas of computer networks. More studies are required to inculcate the idea of collective intelligence in various areas of computer networks. It is therefore very 
promising to identify opportunities for integration, and inculcation of collective intelligence methodologies and technologies into networking. This would require judiciously considering the various activities in a computer network for this purpose. Such an effort would finally aim at remodeling of computer networks in context of collective intelligence.

Chapter 6 by J. Abdullah presents QoS routing algorithm for mobile ad hoc networks (MANET) with specialized encoding, initialization, and route search using genetic algorithm (GA). The objective is to find the best QoS route in order optimize the design of MANET routing protocols. This NP-hard problem is often highly constrained such that random initialization and standard genetic operators usually generate infeasible networks. Another complication is that the fitness function involves calculating all node reliability of the routes, a calculation that is computationally expensive. Therefore, it is imperative that the search balances the need to thoroughly explore the boundary between feasible and infeasible networks, along with calculating fitness on only the most promising candidate routes. The algorithm results are compared to the result of standard MANET protocols without the GA search algorithm.

Chapter 7 by S. Sarwari and S. Rao considers a thermal network, which is a network where the flow functionality of a node depends upon its temperature. This model is inspired by several types of real-life networks, and generalizes some conventional network models wherein nodes have fixed capacities, and the problem is to maximize the flow through the network. In a thermal network, the temperature of a node increases as traffic moves through it, and nodes may also cool spontaneously over time, or by employing cooling packets. The problems of maximizing the flow from a source to a sink are analyzed for both these cases, for a holistic view with respect to the single-source-singlesink dynamic flow problem in a thermal network. The chapter also studies certain properties that a thermal network exhibits, and gives closed-form solutions for the maximum flow that can be achieved through such a network.

Chapter 8 by M.A.J. Sethi, F.A. Hussin, and N.H. Hamid gets inspiration from biological brain fault tolerant techniques to make Network on Chip (NoC) communication reliable as the biological brain is highly robust and fault tolerant. The brain tries to work properly, even if some neurons, synapses, or some other part of the brain is damaged. Synaptogenesis and sprouting are two biological brain techniques used in this chapter to implement the self-adapt and self-heal concepts in NoC.

Finally, Chapter 9 by M.A.J. Sethi, F.A. Hussin, and N.H. Hamid introduces two broad categories of QoS parameters in NoC: guaranteed throughput connection service (GT) and best-effort connection service (BE). The bio-inspired algorithm is implemented using these connection setups. The GT connections are implemented using time division multiplexing (TDM). TDM connections helps divide the bandwidth of interconnect among multiple connections using slots. The BE connections are implemented using packet switching. In packet switching, routers decide at which direction the packet should be sent based on the situation of neighbor's routers, interconnects, and routing table. In this chapter, the bio-inspired NoC algorithm using GT connection is discussed.

\section{Remarkable Features of the Book}

This book has the following remarkable features:

- An advanced reference on Nature-Inspired Networks

- A technique guide on Nature-Inspired Networks

- State-of-the-art solutions used in the field of Nature-Inspired Networks

- Illustrations enable easy reading and comprehension

- Based on rigorous interdisciplinary approaches in which theoretical contributions are formally stated and justified, and practical applications based on their firm formal basis.

The book serves as a comprehensive and essential reference on $\mathrm{NiN}$ and is intended as a textbook for senior undergraduate- and graduate-level courses. It can also be used as a supplementary textbook for undergraduate courses. The book is a useful resource for students and researchers to learn NiN. In addition, it will be valuable to professionals from both academia and industry and generally has instant appeal to people who would like to contribute to NiN technologies.

We highly hope you enjoy reading the book.

\section{Reviews and Testimonials}

"“Nature-inspired" includes, roughly speaking, "bioinspired"+"physical-inspired"+"social-inspired"+ and so on. This book contains highly original contributions about how nature is going to shape networking systems of the future".

\section{- CRC Press}

"The book will usefully serve as a technical guide and reference material for engineers, scientists, practitioners, and researchers by providing them with state-of-the-art research results and future opportunities and trends. To the best of my knowledge, this is one of the first books that presents achievements and findings of NiN research. These 
make the book unique and, in more than one respect, a truly valuable source of information that may be considered a landmark in the progress of NiN".

- Nguyen Thanh Tung, Vietnam National University - Hanoi

\section{References}

[1] P.C. VINH(2018) Nature-Inspired Networking: Theory and Applications (CRC Press), 1st ed, 364 pages, ISBN:9781498761505. 